\title{
CONSTRUCTION OF AN ORTHONORMAL COMPLEX MULTIRESOLUTION ANALYSIS
}

\author{
Liying Wei and Thierry Blu \\ Department of Electronic Engineering, The Chinese University of Hong Kong, \\ Shatin, N.T., Hong Kong
}

\begin{abstract}
We design two complex filters $\{h[n], g[n])\}$ for an orthogonal filter bank structure based on two atom functions $\left\{\rho_{0}^{\alpha}(t), \rho_{1 / 2}^{\alpha}(t)\right\}$, such that: 1) they generate an orthonormal multiwavelet basis; 2) the two complex conjugate wavelets are Hilbert wavelets, i.e., their frequency responses are supported either on positive or negative frequencies; and 3) the two scaling functions are real. The developed complex wavelet transform (CWT) is non-redundant, nearly shiftinvariant, and distinguishable for diagonal features. The distinguishability in diagonal features is demonstrated by comparison with real discrete wavelet transform.
\end{abstract}

Index Terms - Non-redundant, orthonormal complex multiresolution analysis

\section{INTRODUCTION}

Though real wavelet transform has widely used in signal and image processing, it suffers from two main problems: 1) shift invariance; and 2) poor directional selectivity. Complex wavelet transform (CWT) is developed to overcome these two problems [1,2]. But current CWT introduces some redundancy into the transform, such as the single-tree CWT has redundancy $2^{J}$ with $J$ scale level [1] and the dual-tree DTCWT has redundancy 4 [2] for two dimensional images. However, the redundancy complicates such applications as image/video compression where a parsimonious signal representation is critical. To address this problem, some non-redundant CWTs have been proposed [3-5]. But [3,4] neither provide explicit filters nor discuss the complex multiresolution analysis (MRA). Paper [5] proposed a pair of complex filters where the wavelets are Hilbert functions, but the complex MRA is not orthonormal, i.e., the frequency responses of the wavelets and scaling functions are overlapped, which makes hard distinguish the diagonal components for images.

In this paper, based on the same orthconjugate filter bank structure shown in Fig 1 as [5], we aim to develop a new complex wavelet transform which is non-redundant for real input signals, nearyly shift-invariant and distinguishable in the diagonal features. In detail, our objective is to

This work was supported by the General Research Fund CUHK410209 from the Hong Kong Research Grant Council. design complex filters $\{h[n], g[n])\}$ by two atom functions $\left\{\rho_{0}^{\alpha}(t), \rho_{1 / 2}^{\alpha}(t)\right\}$, such that: 1) they generate an orthonormal complex multiresolution analysis; 2 ) the two complex conjugate wavelets are Hilbert wavelets that offer shift-invariance and directivity; and 3) the two scaling functions are real that preserve the polynomial trends for signals between scales. Here, $\left\{\rho_{0}^{\alpha}(t), \rho_{1 / 2}^{\alpha}(t)\right\}$ are such functions whose frequency responses are $\hat{\rho}_{0}^{\alpha}(\omega)=\frac{1}{|\omega|^{\alpha+1}}$ and $\hat{\rho}_{1 / 2}^{\alpha}(\omega)=\frac{-j \operatorname{sgn}(\omega)}{|\omega|^{\alpha+1}}$, where $\alpha$ is a degree parameter and $\operatorname{sgn}(\omega)$ is the sign function with 1 for $\omega>0,-1$ for $\omega<0$, and $\frac{1}{2}$ for $\omega=0$. The Hilbert wavelet is a complex function whose imaginary part is the Hilbert transform of the real part, i.e., its support of the frequency respose is either in positive or in negative.

Because an orthogonal filter bank is only the necessary condition to generate an orthonormal complex MRA [6], so we will start from the sufficient condition: firstly construct the orthonormal complex MRA and then determine the filters.

\section{CONSTRUCTION OF AN ORTHONORMAL COMPLEX MULTIRESOLUTION ANALYSIS}

Let $\Re\{\cdot\}, \Im\{\cdot\},[\cdot]^{*}$ and $\widetilde{[\cdot]}$ be real, imaginary, complex conjugate and synthesis filter operators, respectively. In Fig. 1, $u[n]=\left\{\frac{1}{\sqrt{2}}, \frac{j}{\sqrt{2}}\right\}$ is an orthonormal filter derived from the Haar filter by frequency shifting; $x[n], y[n]$ and $w[n]$ denote the input, lowpass output for iteration and highpass output (wavelet coefficients), respectively. There exist: 1) $w[n]=$ $\sum_{k} g[k-4 n] x[k]$;2) $y[n]=\sum_{k, l} 2 \Re\{u[2 k-n] h[l-4 k]\} x[l]$. Our objective is to design the complex filters $\{h[n], g[n]\}$ by two atom functions $\left\{\rho_{0}^{\alpha}(t), \rho_{1 / 2}^{\alpha}(t)\right\}$, such that $\{h[n], g[n]\}$ generates an orthonormal complex multiresolution analysis.

\subsection{Complex multiresolution analysis}

Let $\left\{\phi_{0}(t), \phi_{1}(t)\right\}$ be the scaling functions and $\left\{\psi(t), \psi^{*}(t)\right\}$ be the complex conjugate wavelets. Define subspaces $V_{j}=$ $\operatorname{Span}_{n \in \mathbb{Z}}\left\{\phi_{0}\left(2^{j} t-n\right), \phi_{1}\left(2^{j} t-n\right)\right\}, W_{j}=\operatorname{Span}_{n \in \mathbb{Z}}\left\{\psi\left(2^{j} t-\right.\right.$ $n)\}$ and $W_{j}^{*}=\operatorname{Span}_{n \in \mathbb{Z}}\left\{\psi^{*}\left(2^{j} t-n\right)\right\}$. The complex multiresolution analysis states that: 1) $\cdots \subset V_{-1} \subset V_{0} \subset$ $V_{1} \subset \cdots, \overline{\bigcup_{j=-\infty}^{\infty} V_{j}}=L^{2}(\mathbb{R})$ and $\left.\bigcap_{j=-\infty}^{\infty} V_{j}=\{0\} ; 2\right)$ $V_{j}=V_{j-1} \oplus W_{j-1} \oplus W_{j-1}^{*}$, where $\oplus$ denotes the Kronecker direct sum; and 3) any square integrable real function 


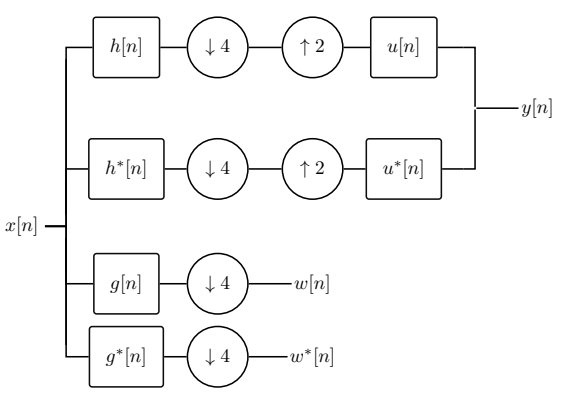

(a) Analysis filter bank

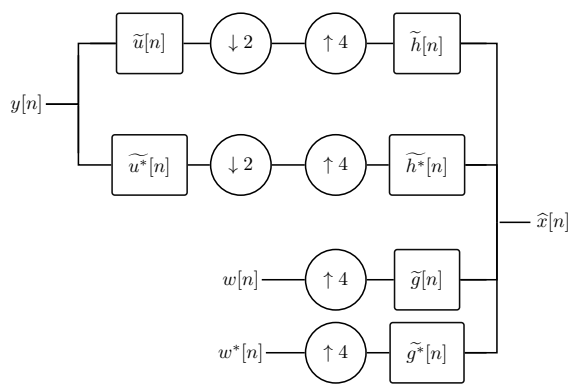

(b) Synthesis filter bank

Fig. 1. The orthconjugate filter bank structure for 1D non-redundant complex wavelet transform (NRCWT) implementation.

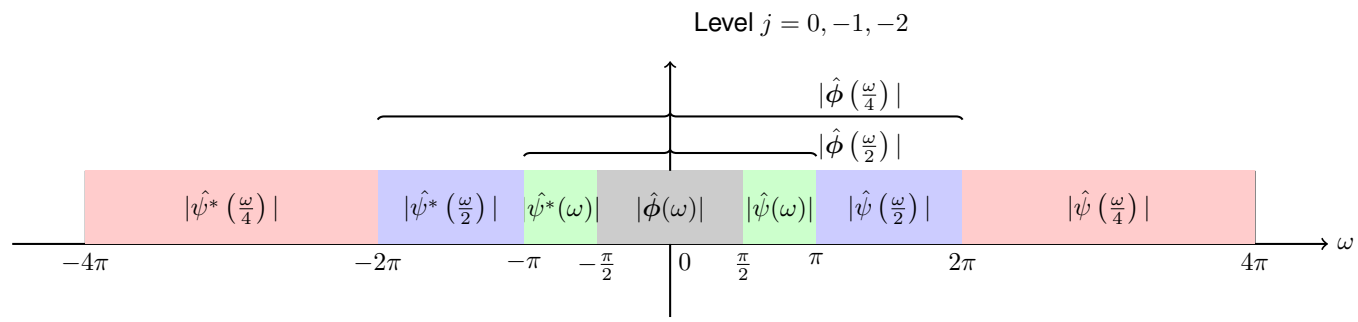

Fig. 2. Frequency-domain energy localization of the orthonormal multiwavelet basis $\left\{2^{j / 2} \phi_{i}\left(2^{j} t-n\right), 2^{j / 2} \psi\left(2^{j} t-\right.\right.$ $\left.n), \psi^{*}\left(2^{j} t-n\right)\right\}$, where $\phi_{i}\left(2^{j} t-n\right)$ are real functions whose frequency responses are supported within $\left[-2^{j-1} \pi, 2^{j-1} \pi\right]$, while $\psi\left(2^{j} t-n\right)$ are Hilbert functions whose frequency responses are supported in the positive frequencies $\left[2^{j-1} \pi, 2^{j} \pi\right]$.

$f(\cdot) \in V_{j}$, there exists $f(2 \cdot) \in V_{j+1}$. The complex multiresolution analysis implies both the scaling functions and the wavelets satisfy a matrix-dilation equation,

$$
\begin{aligned}
& {\left[\begin{array}{c}
\phi_{0}\left(\frac{t}{2}\right) \\
\phi_{1}\left(\frac{t}{2}\right)
\end{array}\right]=\sqrt{2} \sum_{l} M[l]\left[\begin{array}{l}
\phi_{0}(t-l) \\
\phi_{1}(t-l)
\end{array}\right],} \\
& {\left[\begin{array}{c}
\psi\left(\frac{t}{2}\right) \\
\psi^{*}\left(\frac{t}{2}\right)
\end{array}\right]=\sqrt{2} \sum_{l} N[l]\left[\begin{array}{c}
\phi_{0}(t-l) \\
\phi_{1}(t-l)
\end{array}\right],}
\end{aligned}
$$

where $M[l]=\sqrt{2}\left[\begin{array}{cc}\Re\{h[2 l]\} & \Re\{h[2 l-1]\} \\ -\Im\{h[2 l]\} & -\Im\{h[2 l-1]\}\end{array}\right]$ and $N[l]=\left[\begin{array}{cc}g[2 l] & g[2 l-1] \\ g^{*}[2 l] & g^{*}[2 l-1]\end{array}\right] \triangleq\left[\begin{array}{cc}g_{0}[l] & g_{1}[l] \\ g_{0}^{*}[l] & g_{1}^{*}[l]\end{array}\right]$. Define the vector functions $\boldsymbol{\phi}(t)=\left[\begin{array}{c}\phi_{0}(t) \\ \phi_{1}(t)\end{array}\right], \boldsymbol{\psi}(t)=\left[\begin{array}{c}\psi(t) \\ \psi^{*}(t)\end{array}\right]$. Let $\hat{\phi}(\omega)$ and $\hat{\psi}(\omega)$ be their Fourier transforms. Then the matrix-dilation equations in the frequency domain can be represented as

$$
\begin{aligned}
& \hat{\phi}(\omega)=\frac{\sqrt{2}}{2} \boldsymbol{M}\left(e^{j \frac{\omega}{2}}\right) \hat{\boldsymbol{\phi}}\left(\frac{\omega}{2}\right), \\
& \hat{\boldsymbol{\psi}}(\omega)=\frac{\sqrt{2}}{2} \boldsymbol{N}\left(e^{j \frac{\omega}{2}}\right) \hat{\boldsymbol{\phi}}\left(\frac{\omega}{2}\right),
\end{aligned}
$$

where $\boldsymbol{M}\left(e^{j \omega}\right)=\sum_{l} M[l] e^{-j l \omega}$ and $\boldsymbol{N}\left(e^{j \omega}\right)=\sum_{l} N[l] e^{-j l \omega}$ are called the refinement matrices.
The complex MRA is orthonormal if and only if:

- $\left\{\phi_{0}(t-n), \phi_{1}(t-n)\right\}$ forms a basis for the subspace $V_{0}$, i.e., $\langle\boldsymbol{\phi}(t), \boldsymbol{\phi}(t-n)\rangle=\boldsymbol{I}_{2} \delta_{n 0}$, where $\boldsymbol{I}_{2}$ is a twodimensional identity matrix and $\delta$ is the Kroneck delta function;

- $\psi(t-n)$ forms a basis for the subspace $W_{0}$ and $\psi^{*}(t-n)$ forms a basis for the subspace $W_{0}^{*}$, i.e., $\langle\boldsymbol{\psi}(t), \boldsymbol{\psi}(t-n)\rangle=\boldsymbol{I}_{2} \delta_{n 0} ;$

- $\left\{\psi(t-n), \psi^{*}(t-n)\right\}$ are orthogonal to $\left\{\phi_{0}(t-\right.$ $\left.n), \phi_{1}(t-n)\right\}$, i.e., $\langle\boldsymbol{\phi}(t), \boldsymbol{\psi}(t-n)\rangle=\mathbf{0}_{2}$ where $\mathbf{0}_{2}$ is a two-dimensional zero matrix.

\subsection{Problem}

In this paper, our objective is to determine $\{h[n], g[n]\}$ or equivalently $\left\{\boldsymbol{M}\left(e^{j \omega}\right), \boldsymbol{N}\left(e^{j \omega}\right)\right\}$ by $\left\{\rho_{0}^{\alpha}(t), \rho_{1 / 2}^{\alpha}(t)\right\}$, such that: 1) the complex multiresolution analysis is orthonormal; 2) the wavelets are Hilbert functions; and 3) the scaling functions are real.

Note that though $\phi_{0}(t)$ and $\phi_{1}(t)$ are orthogonal each other, their freqeuncy responses might be overlapped. Assume the scaling functions $\hat{\phi}(\omega)$ are supported within $\left[-\frac{\pi}{2}, \frac{\pi}{2}\right]$, while the wavelet $\hat{\psi}(\omega)$ is supported within $\left[\frac{\pi}{2}, \pi\right]$. Fig. 2 plotts the frequency enery localizations of the orthonormal 


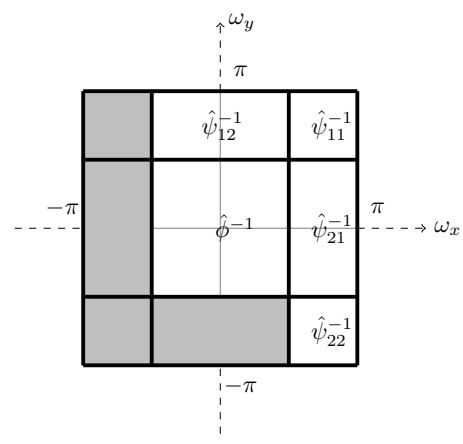

Fig. 3. Frequency-domain energy localization of the orthonormal multiwavelet basis $\left\{2^{j / 2} \phi_{i}\left(2^{j} t-n\right), 2^{j / 2} \psi\left(2^{j} t-\right.\right.$ $\left.n), \psi^{*}\left(2^{j} t-n\right)\right\}$ after 2D NRCWT. $\hat{\phi}^{-1}$ is the Fourier transform of the tensor scaling functions by $\phi_{i}\left(2^{j} t-n\right)$ at scale $j=1$, while $\hat{\psi}_{p q}^{-1}$ are the Fourier transorm of the tensor wavelets at scale $j=-1$ for $p, q=1,2$. The gray parts are the complex conjugates of the white parts in the border.

multiwavelet basis where the wavelets are Hilbert functions. In the figure, $\hat{\phi}(\omega)$ represents both $\hat{\phi}_{0}(\omega)$ and $\hat{\phi}_{1}(\omega)$. It is clear that as the scale $j$ decreases by 1 , the frequency supports of the scaling functions and wavelets are double.

By implementating 2D NRCWT and removing the complex conjugate highpass branches, four separately complex wavelets and one real scaling function are generated. Fig. 3 plotts the the frequency energy localizations of these 2D complex wavelet basis functions, where the gray color parts corresponding to the removed complex conjugate wavelets. Because of the one-sided frequency support of the Hilbert wavelet, this filter bank provides distinct basis functions in vertical $\left(\hat{\psi}_{21}^{-1}\right)$, horizontal $\left(\hat{\psi}_{12}^{-1}\right),+45^{\circ}$ degree $\left(\hat{\psi}_{11}^{-1}\right)$ and $-45^{\circ}$ degree $\left(\hat{\psi}_{22}^{-1}\right)$ subbands.

\subsection{Formulation}

Define $\rho^{\alpha}(t)=\left[\begin{array}{c}\rho_{0}^{\alpha}(t-n) \\ \rho_{1 / 2}^{\alpha}(t-n)\end{array}\right]$. Our objective is to find two coefficinet matrices $C_{n}$ and $D_{n}$ that satisfy

$\boldsymbol{\phi}\left(\frac{t}{2}\right)=\sum_{n} C_{n} \boldsymbol{\rho}^{\alpha}\left(\frac{t}{2}-n\right), \boldsymbol{\psi}\left(\frac{t}{2}\right)=\sum_{n} D_{n} \boldsymbol{\rho}^{\alpha}(t-n)$.

such that $\{h[n], g[n]\}$ generate an orthonormal complex multiresolution analysis (OCMA) with $\boldsymbol{\psi}\left(\frac{t}{2}\right)$ Hilbert functions and $\phi\left(\frac{t}{2}\right)$ are real. Taking the Fourier transform, we have

$$
\hat{\boldsymbol{\phi}}(\omega)=\boldsymbol{C}\left(e^{j \omega}\right) \hat{\boldsymbol{\rho}}^{\alpha}(\omega), \quad \hat{\boldsymbol{\psi}}(\omega)=2^{\alpha} \boldsymbol{D}\left(e^{j \frac{\omega}{2}}\right) \hat{\boldsymbol{\rho}}^{\alpha}(\omega),
$$

where $\boldsymbol{C}\left(e^{j \omega}\right)=\sum_{n} C_{n} e^{-j n \omega}, \boldsymbol{D}\left(e^{j \omega}\right)=\sum_{n} D_{n} e^{-j n \omega}$ and $\hat{\boldsymbol{\rho}}^{\alpha}(\omega)=\left[\begin{array}{c}\rho_{0}^{\alpha}(\omega) \\ \rho_{1 / 2}^{\alpha}(\omega)\end{array}\right]$. Relating to (2), there exist

$$
\begin{aligned}
& \boldsymbol{M}\left(e^{j \omega}\right)=2^{-(\alpha+1 / 2)} \boldsymbol{C}\left(e^{j 2 \omega}\right) \boldsymbol{C}\left(e^{j \omega}\right)^{-1}, \\
& \boldsymbol{N}\left(e^{j \omega}\right)=\frac{\sqrt{2}}{2} \boldsymbol{D}\left(e^{j \omega}\right) \boldsymbol{C}\left(e^{j \omega}\right)^{-1}
\end{aligned}
$$

where $[\cdot]^{-1}$ denotes the inverse operator.

The orthonormality of the complex multiresolution analysis in the frequency domain is equivalent that [7]

$$
\begin{aligned}
& \frac{1}{2} \boldsymbol{I}_{2}=\boldsymbol{C}\left(e^{j \omega}\right) \boldsymbol{R}\left(e^{j \omega}\right) \boldsymbol{C}\left(e^{j \omega}\right)^{H}, \\
& 2 \boldsymbol{I}_{2}=\boldsymbol{D}\left(e^{j \omega}\right) \boldsymbol{R}\left(e^{j \omega}\right) \boldsymbol{D}\left(e^{j \omega}\right)^{H} \\
& \quad+\boldsymbol{D}\left(-e^{j \omega}\right) \boldsymbol{R}\left(-e^{j \omega}\right) \boldsymbol{D}\left(-e^{j \omega}\right)^{H}, \\
& \mathbf{0}_{2}=\boldsymbol{C}\left(e^{j 2 \omega}\right) \boldsymbol{R}\left(e^{j \omega}\right) \boldsymbol{D}\left(e^{j \omega}\right)^{H} \\
& \quad+\boldsymbol{C}\left(e^{j \omega \omega}\right) \boldsymbol{R}\left(-e^{j \omega}\right) \boldsymbol{D}\left(-e^{j \omega}\right)^{H},
\end{aligned}
$$

where $\boldsymbol{R}\left(e^{j \omega}\right)$ is the autocorrelation matrix-filter:

$\boldsymbol{R}\left(e^{j \omega}\right)=\left[\begin{array}{cc}\sum_{k} \frac{1}{|\omega+2 k \pi|^{2(\alpha+1)}} & j \sum_{k} \frac{\operatorname{sgn}(\omega+2 \mathrm{k} \pi)}{|\omega+2 k \pi|^{2(\alpha+1)}} \\ -j \sum_{k} \frac{\operatorname{sgn}(\omega+2 \mathrm{k} \pi)}{|\omega+2 k \pi|^{2(\alpha+1)}} & \sum_{k} \frac{1}{|\omega+2 k \pi|^{2(\alpha+1)}}\end{array}\right]$.

Our objective is to obtain $\left\{\boldsymbol{C}\left(e^{j \omega}\right), \boldsymbol{D}\left(e^{j \omega}\right)\right\}$ by solving (4).

\subsection{Construction of orthonormal complex MRA}

By using spectral decomposition and linear algebra operation to (4), we get the coefficient matrices with

$$
\begin{aligned}
& \boldsymbol{C}\left(e^{j \omega}\right)=\left[\begin{array}{cc}
e^{j \beta_{3}} & 0 \\
0 & e^{j \beta_{4}}
\end{array}\right]\left[\begin{array}{cc}
\cos \theta_{2} & -\sin \theta_{2} \\
\sin \theta_{2} & \cos \theta_{2}
\end{array}\right] \\
& \times\left[\begin{array}{cc}
\frac{2}{\sqrt{a\left(e^{j \omega}\right)}} & \frac{j 2}{\sqrt{a\left(e^{j \omega}\right)}} \\
\frac{2}{\sqrt{a\left(e^{-j \omega}\right)}} & \frac{-j 2}{\sqrt{a\left(e^{-j \omega}\right)}}
\end{array}\right] \\
& \boldsymbol{D}\left(e^{j \omega}\right) \\
& =e^{-j \omega}\left[\begin{array}{cc}
\cos \theta_{1} & \sin \theta_{1} \\
-\sin \theta_{1} & \cos \theta_{1}
\end{array}\right]\left[\begin{array}{cc}
e^{j \beta_{1}} & 0 \\
0 & e^{j \beta_{2}}
\end{array}\right] \times \\
& {\left[\begin{array}{cc}
\sqrt{\frac{1}{a\left(e^{j \omega}\right)}-\frac{2^{-2(\alpha+1)}}{a\left(e^{j 2 \omega}\right)}} & j \sqrt{\frac{1}{a\left(e^{j \omega}\right)}-\frac{2^{-2(\alpha+1)}}{a\left(e^{j 2 \omega}\right)}} \\
\sqrt{\frac{1}{a\left(e^{-j \omega}\right)}-\frac{2^{-2(\alpha+1)}}{a\left(e^{-j 2 \omega}\right)}} & -j \sqrt{\frac{1}{a\left(e^{-j \omega}\right)}-\frac{2^{-2(\alpha+1)}}{a\left(e^{-j 2 \omega}\right)}}
\end{array}\right],}
\end{aligned}
$$

where $\beta_{i}$ and $\theta_{j}$ are real parameters of $\omega$. The corresponding wavelets and scaling functions can be also represented by

$$
\begin{aligned}
& {\left[\begin{array}{l}
\widehat{\phi}_{0}(\omega) \\
\widehat{\phi}_{1}(\omega)
\end{array}\right]=\boldsymbol{C}\left(e^{j \omega}\right) \hat{\boldsymbol{\rho}}^{\alpha}(\omega)} \\
& =\left[\begin{array}{l}
\left(\frac{2 \cos \theta_{2}}{\sqrt{a\left(e^{j \omega}\right)}} \frac{1+\operatorname{sgn}(\omega)}{|\omega|^{\alpha+1}}-\frac{2 \sin \theta_{2}}{\sqrt{a\left(e^{-j \omega}\right)}} \frac{1-\operatorname{sgn}(\omega)}{|\omega|^{\alpha+1}}\right) e^{j \beta_{3}} \\
\left(\frac{2 \sin \theta_{2}}{\sqrt{a\left(e^{j \omega}\right)}} \frac{1+\operatorname{sgn}(\omega)}{|\omega|^{\alpha+1}}+\frac{2 \cos \theta_{2}}{\sqrt{a\left(e^{-j \omega}\right)}} \frac{1-\operatorname{sgn}(\omega)}{|\omega|^{\alpha+1}}\right) e^{j \beta_{4}}
\end{array}\right],
\end{aligned}
$$




$$
\begin{aligned}
& {\left[\begin{array}{c}
\widehat{\psi}(\omega) \\
\widehat{\psi}^{*}(-\omega)
\end{array}\right]=2^{\alpha} \boldsymbol{D}\left(e^{j \frac{\omega}{2}}\right) \hat{\boldsymbol{\rho}}^{\alpha}(\omega)} \\
& =\left[\begin{array}{cc}
\cos \theta_{1} & \sin \theta_{1} \\
-\sin \theta_{1} & \cos \theta_{1}
\end{array}\right]\left[\begin{array}{cc}
e^{j \beta_{1}} & 0 \\
0 & e^{j \beta_{2}}
\end{array}\right] \\
& \times\left[\begin{array}{c}
2^{\alpha} \sqrt{\frac{1}{a\left(e^{j \frac{\omega}{2}}\right)}-\frac{2^{-2(\alpha+1)}}{a\left(e^{j \omega}\right)}} \frac{1+\operatorname{sgn}\left(\frac{\omega}{2}\right)}{|\omega|^{\alpha+1}} e^{-j \frac{\omega}{2}} \\
2^{\alpha} \sqrt{\frac{1}{a\left(e^{-j \frac{\omega}{2}}\right)}-\frac{2^{-2(\alpha+1)}}{a\left(e^{-j \omega}\right)}} \frac{1-\operatorname{sgn}\left(\frac{\omega}{2}\right)}{|\omega|^{\alpha+1}} e^{-j \frac{\omega}{2}}
\end{array}\right] .
\end{aligned}
$$

Let $\theta_{1}=\beta_{1}=\beta_{2}=\beta_{4}=0, \theta_{2}=\frac{\pi}{4}$ and $e^{j \beta_{3}(\omega)}=$ $\operatorname{sgn}(\omega) \mathrm{e}^{\mathrm{j} \frac{\omega}{2}}$, we get a pair of real scaling functions, a pair of Hilbert wavelets; particularly, we also obtain a highpass filter $G\left(e^{j \omega}\right)=G_{0}\left(e^{j 2 \omega}\right)+e^{-j \omega} G_{1}\left(e^{j 2 \omega}\right)$ which is only supported in the positive frequencies.

\subsection{Complex filters}

Applying (3), we obtain $\boldsymbol{M}\left(e^{j \omega}\right)$ and $\boldsymbol{N}\left(e^{j \omega}\right)$; then by using the polyphase filters [8],

$$
\left[\begin{array}{c}
F_{0}(z) \\
F_{1}(z)
\end{array}\right]=\boldsymbol{M}\left(z^{2}\right)\left[\begin{array}{c}
1 \\
z^{-1}
\end{array}\right],\left[\begin{array}{l}
F_{2}(z) \\
F_{3}(z)
\end{array}\right]=\boldsymbol{N}\left(z^{2}\right)\left[\begin{array}{c}
1 \\
z^{-1}
\end{array}\right]
$$

the two complex filters in the $\mathrm{z}$-domain are solved,

$$
H(z)=\frac{\sqrt{2}}{2}\left[F_{0}(z)-j F_{1}(z)\right], \quad G(z)=F_{2}(z) .
$$

Figure 4 plots the specifical complex filters, scaling functions and wavelets when $\alpha=4.5$. It is noticable that 1) $\left|H\left(e^{j \omega}\right)\right|$ is asymmetric and continuous in $\omega \in\left(-\frac{\pi}{4}, \frac{\pi}{4}\right)$, while $\left|G\left(e^{j \omega}\right)\right|$ is almost supported in $\left.\omega \in\left(\frac{\pi}{2}, \pi\right) ; 2\right)$ the scaling functions $\widehat{\phi}_{i}(\omega)$ have the same magnitudes and are supported in $\omega \in$ $(-\pi, \pi)$; and 3) the wavelet $\widehat{\psi}(\omega)$ is in $\omega \in(\pi, 2 \pi)$.
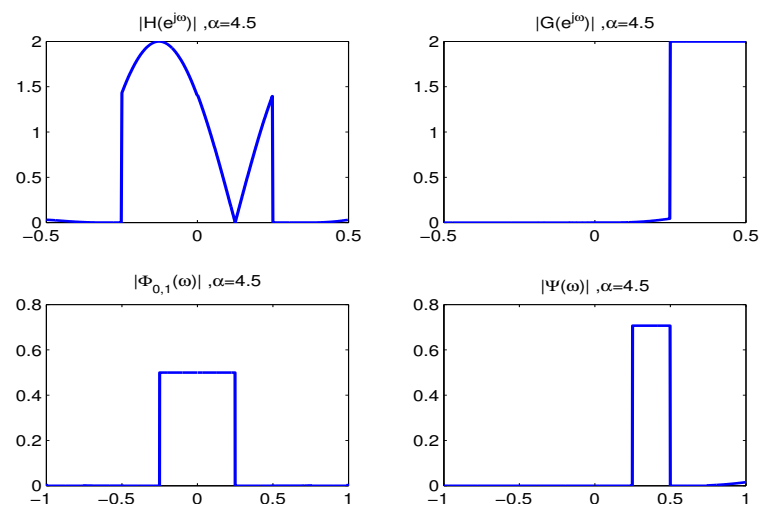

Fig. 4. The frequency responses of the complex filters $\left\{H\left(e^{j \omega}\right), G\left(e^{j \omega}\right)\right\}(8)$ with $z=e^{j \omega}$, the real scaling functions (6) and the Hilbert wavelets (7) when $\alpha=4.5$. The $\mathrm{x}$-label is $\frac{\omega}{2 \pi}$.

\section{PROPERTIES OF NRCWT}

The distinguishability of our NRCWT in diagonal features provided by $\left\{H\left(e^{j \omega}\right), G\left(e^{j \omega}\right)\right\}$ is demonstrated by Fig. 5 for 'Architecture.jpg'. In the Fig. 5, (a) is the original figure; (b) is the discrete wavelet transform (DWT) figure taken from the diagonal subband of the classical two-band perfect reconstruction filter bank at scale level 3, where the real, normalized fractional $(\alpha, \tau)$-B-spline filters (FBF) with $\alpha=4.5$ and $\tau=0$ are used [9]; (c) and (d) are the complex wavelet transform (CWT) figures taken from our 2D NRCWT ' $+45^{\circ}$ ' diagonal' subband and ' $-45^{\circ}$ ' diagonal' subband at scale leve 2 with $\alpha=4.5$. Observing these figures, we can see that both the ' $+45^{\circ}$ ' line features in (c) and the ' $-45^{\circ}$ ' line features in (d) are highly enhanced and could be easily extracted; however, in subfig (b), these two directionally line features are tangled each other, which makes hard split and extract the $'+45^{\circ}$ ' directional features from ' $-45^{\circ}$ ' directional features.

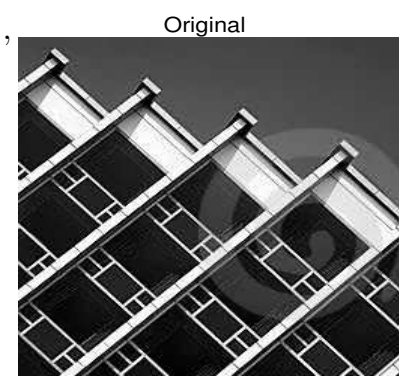

(a)

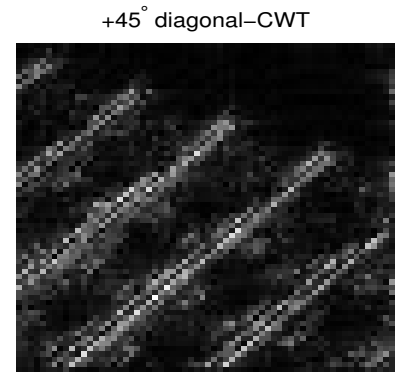

(c)

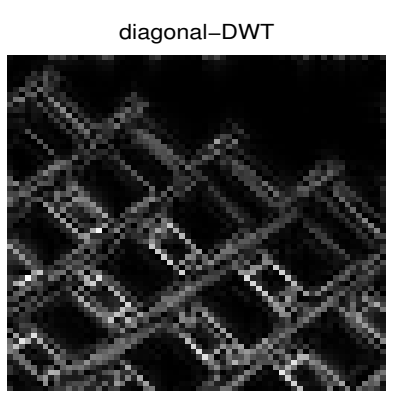

(b)

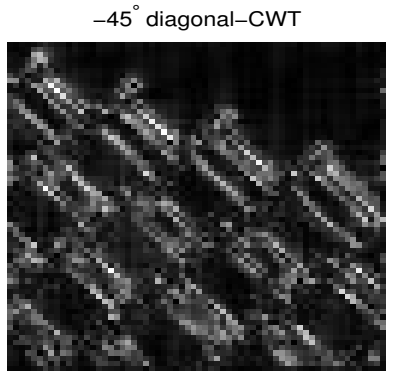

(d)
Fig. 5. Demonstration of directivities of 2D NRCWT. (a) - original image, (b) - image from the diagonal subband of classical two-band perfect reconstruction filter bank with real, normalized fractional $(\alpha, \tau)$-B-spline filters (FBF) for $\alpha=4.5$ and $\tau=0$, (c) and (d) - images from the diagonal subbands of our 2D NRCWT: ' $+45^{\circ}$ diagonal' subband and ' $-45^{\circ}$ diagonal' subband. The line features along the positive and negative diagonal directions can be easily distinguished, splitted and extracted from (c) and (d), respectively. 


\section{REFERENCES}

[1] J. Magarey and N. Kingsbury, "Motion estimation using a complex-valued wavelet transform," IEEE Trans. Signal Process., vol. 46, no. 4, pp. 1069-1084, Apr. 1998.

[2] N. Kingsbury, "Image processing with complex wavelets," Phil. Trans. R. Soc. Lond. A, vol. 357, no. 1760, pp. 2543-2560, Sep. 1999.

[3] F. C. A. Fernandes, R. L. C. van Spaendonck, and C. S. Burrus, "A new framework for complex wavelet transforms," IEEE Trans. Signal Process., vol. 51, no. 7, pp. 1825-1837, Jul. 2003.

[4] R. V. Spaendonck, T. Blu, R. Baraniuk, and M. Vetterli, "Orthogonal Hilbert transform filter banks and wavelets," in Proc. IEEE Int. Conf. Acoust., Speech, Signal Processing (ICASSP'2003), Hong Kong, China, Apr. 6-10 2003, pp. 505-508.

[5] L. Wei and T. Blu, "A new non-redundant complex Hilbert wavelet transform," in Proc. IEEE Stat. Sign. Proc. Workshop (SSP'2012), Ann Arbor, USA, Aug. 58 2012, p. 4.

[6] Q. Jiang, "On the design of multifilter banks and orthonormal multiwavelet bases," IEEE Trans. Signal Process., vol. 46, no. 12, pp. 830-844, Dec. 1998.

[7] S. Yang, Z. Cheng, and H. Wang, "Construction of biorthogonal multiwavelets," J. Math. Anal. Appl., vol. 276, no. 1, pp. 1-12, Dec. 2002.

[8] I. W. Selesnick, "Balanced multiwavelet bases based on symmetric FIR filters," IEEE Trans. Signal Process., vol. 48, no. 1, pp. 184-191, Jan. 2000.

[9] F. Luisier, T. Blu, and M. Unser, "Which wavelet bases are the best for image denoising?" in Proc. SPIE, vol. 5914, Bellingham, WA, Jul. 2005, p. 59140E. 